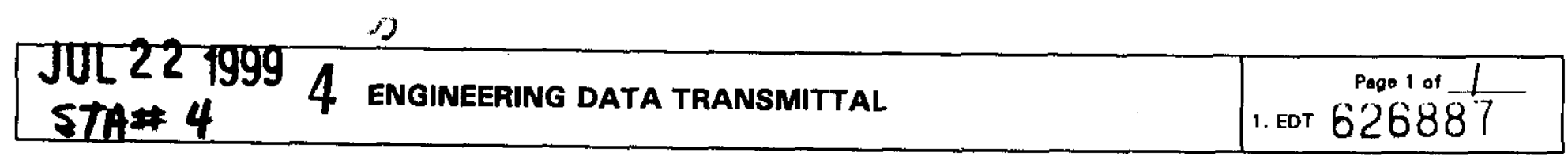

\begin{tabular}{|c|c|c|c|c|c|c|c|c|}
\hline \multicolumn{3}{|c|}{$\begin{array}{l}\text { 2. To: (Receiving Organization) } \\
\text { Distribution }\end{array}$} & \multicolumn{2}{|c|}{$\begin{array}{l}\text { 3. From: (Originating Organization) } \\
\text { Nuclear Safety }\end{array}$} & \multicolumn{4}{|c|}{$\begin{array}{l}\text { 4. Related EDT No.: } \\
\qquad \text { N/A }\end{array}$} \\
\hline \multicolumn{3}{|c|}{$\begin{array}{l}\text { 5. Proj./Prog./Dept./Div.: } \\
\text { Spent Nuclear Fuel Project }\end{array}$} & \multicolumn{2}{|c|}{$\begin{array}{l}\text { 6. Design Authority/ Design Agent/Cog. } \\
\text { Engr.: } \\
\text { S. F. Kessler }\end{array}$} & \multicolumn{4}{|c|}{$\begin{array}{r}\text { 7. Purchase Order } \mathrm{No}: \text { : } \\
\qquad \text { N/A }\end{array}$} \\
\hline \multirow{2}{*}{\multicolumn{5}{|c|}{$\begin{array}{l}\text { 8. Originator Remarks: } \\
\text { N/A }\end{array}$}} & \multicolumn{4}{|c|}{$\begin{array}{r}\text { 9. Equip./Component No:: } \\
\text { N/A }\end{array}$} \\
\hline & & & & & \multicolumn{4}{|c|}{$\begin{array}{r}\text { 10. System/Bldg./Facility: } \\
\text { K Basins }\end{array}$} \\
\hline \multirow{3}{*}{\multicolumn{3}{|c|}{ 11. Receiver Remarks: }} & \multirow{3}{*}{\multicolumn{2}{|c|}{ Document? [] Yes [X] No }} & \multicolumn{4}{|c|}{$\begin{array}{r}\text { 12. Major Assm. Dwg. No.: } \\
\text { N/A }\end{array}$} \\
\hline & & & & & \multicolumn{4}{|c|}{$\begin{array}{l}\text { 13. Permit/Permit Application No.: } \\
\text { N/A }\end{array}$} \\
\hline & & & & & \multicolumn{4}{|c|}{$\begin{array}{l}\text { 14. Required Response Date: } \\
\qquad \text { N/A }\end{array}$} \\
\hline 15. & \multicolumn{4}{|c|}{ DATA TRANSMITTED } & (F) & (G) & $(\mathrm{H})$ & (I) \\
\hline $\begin{array}{l}\text { (A) } \\
\text { Item } \\
\text { No. }\end{array}$ & (B) Document/Drawing No. & $\begin{array}{l}\text { (C) } \\
\text { Sheet } \\
\text { No. }\end{array}$ & $\begin{array}{l}\text { (D) } \\
\text { Rev. } \\
\text { No. }\end{array}$ & (E) Title or Description of Dats Transmitted & $\begin{array}{l}\text { Approval } \\
\text { Desig- } \\
\text { nator }\end{array}$ & $\begin{array}{l}\text { Reason } \\
\text { for Trans- } \\
\text { mittal }\end{array}$ & $\begin{array}{l}\text { Origi- } \\
\text { nator } \\
\text { Dispo- } \\
\text { sition }\end{array}$ & $\begin{array}{l}\text { Receiv- } \\
\text { er } \\
\text { Dispo- } \\
\text { sition }\end{array}$ \\
\hline 1 & SNF-4848 & & 0 & $\begin{array}{l}\text { Complete Misloading of a Mark IV } \\
\text { MCO with Mark 1A Fuel }\end{array}$ & $\mathrm{S}$ & 2 & 1 & 1 \\
\hline & & & & & & & & \\
\hline & & & & & & & & \\
\hline & & & & & & & & \\
\hline & & & & & & & & \\
\hline & & & & & & & & \\
\hline
\end{tabular}

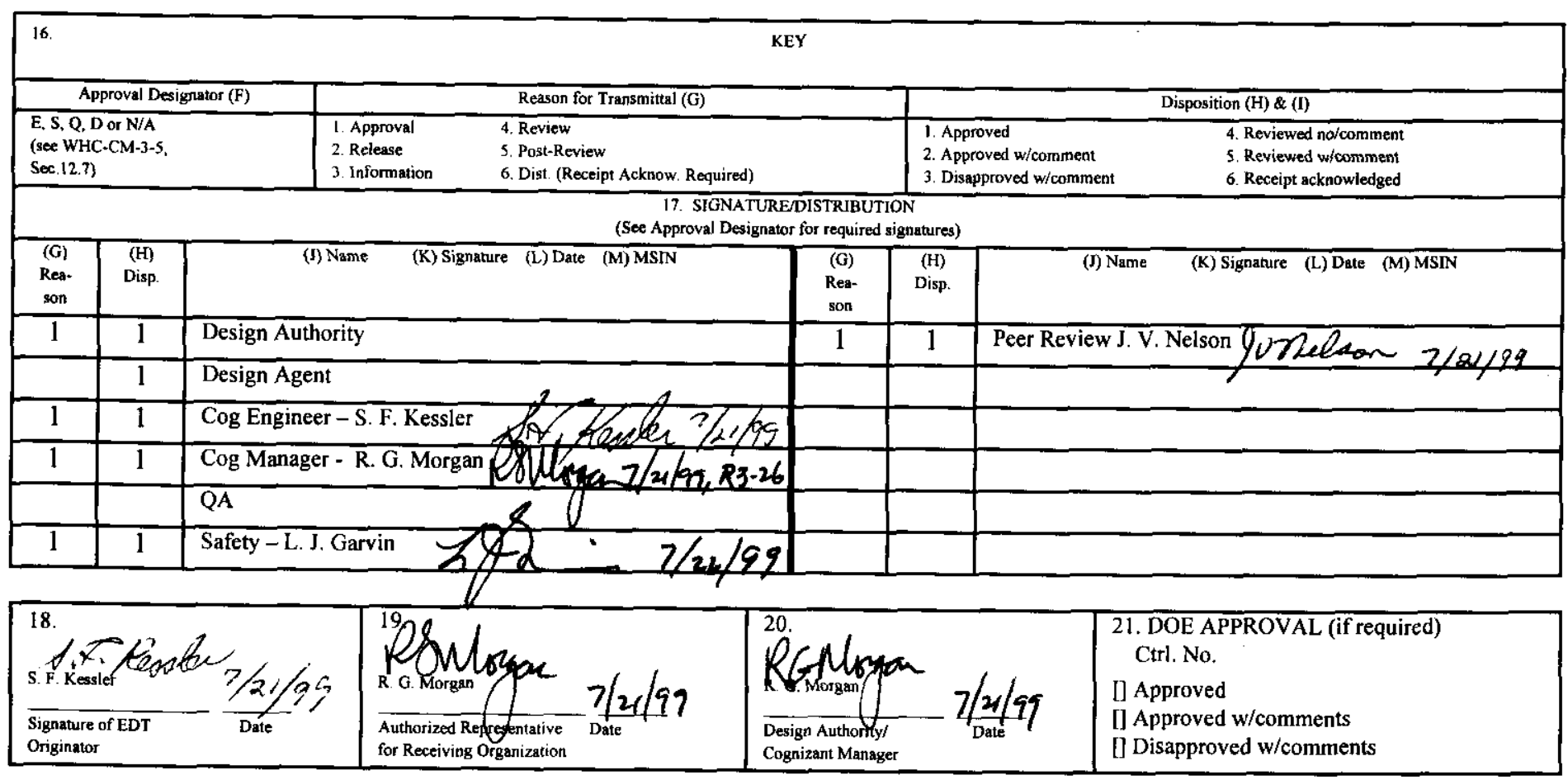

BD-7400-1 72-2 (05/96) GEF097 


\title{
Complete Misloading of a Mark IV MCO with Mark 1A Fuel
}

\author{
S. F. Kessler, J. V. Nelson \\ Fluor Daniel Northwest, Inc., Richland, WA 99352 \\ U.S. Department of Energy Contract DE-AC06-96RL13200 \\ EDT/ECN: 626887 \\ UC: 620 \\ Org Code: 2 F200 \\ B\&R Code: $39 \mathrm{EW} 40400$ \\ Charge Code: $105414 / \mathrm{CB} 80$ \\ Total Pages: 1817 PF $7-22-99$
}

Key Words: Spent Nuclear Fuel Project, K Basins, MCO, Mark 1A and Mark IV Fuel
Abstract: The purpose of this analysis is to determine the worst case scenario for a total misload of a Mark IV MCO with Mark 1A fuel and scrap. This study is not intended to classify any of the components of the baskets

TRADEMARK DISCLAIMER. Reference herein to any specific commercial product, process, or service by trade name, trademark, manufacturer, or otherwise, does not necessarily constitute or imply its endorsement, recommendation, or favoring by the United States Government or any agency thereof or its contractors or subcontractors.

Printed in the United States of America. To obtain copies of this document, contact: Document Control Services, P.O. Box 950, Mailstop H6-08, Richland WA 99352, Phone (509) 372-2420; Fa (509) 376-4989.
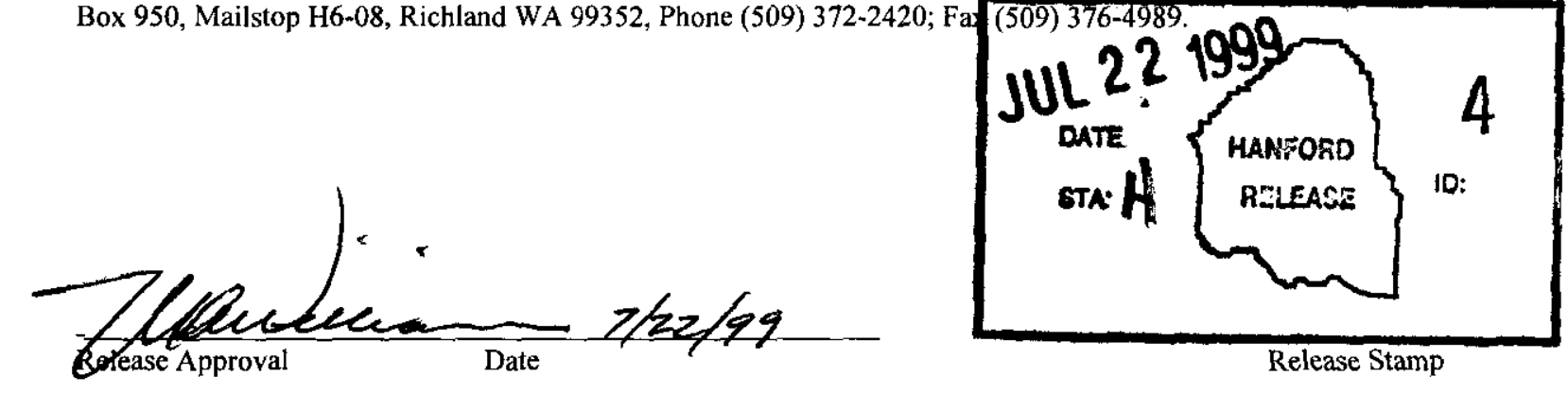

\section{Approved for Public Release}


COMPLETE MISLOADING OF A MARK IV MCO WITH MARK 1A FUEL

Prepared by: $\frac{\text { D. Terslect }}{\text { S. F. Kesster, FDNW Criticality and Shielding }}$ Date: $7 / 21 / 99$

Reviewed by: $\frac{912 \text { Celoor }}{1 . V . \text { Nelson, FDNW Criticality and Shielding }}$ Date: $3 / 20 / 99$ 


\section{COMPLETE MIS-LOADING OF A MARK IV MCO WITH MARK 1A FUEL}

\subsection{Purpose}

The purpose of this analysis is to determine the worst case scenario for a total misload of a Mark IV Multi-Canister Overpack (MCO) with Mark 1A fuel and scrap. This study is not intended to classify any of the components of the baskets.

\subsection{Input Data}

- The model of the MCO was the same as described in HNF-SD-SNF-CSER-005, Criticality Safety Evaluation Report for the MCO, Revision 4.

- Each fuel basket contained 54 Mark 1A fuel assemblies, 20.9 inches in length.

- Each basket contained the central pipe, which is required to lift the basket and to allow insertion of the long process tube for drying at the Cold Vacuum Drying (CVD) Facility.

- The optimization parameters for the scrap were derived in HNF-SD-SNF-CSER-010, Criticality Safety Evaluation Report for the K Basin Fuel Retrieval Subproject, Revision 0.

\subsection{Assumptions}

- The MCO was flooded in the transportation cask.

- The scrap in the scrap baskets was modeled as being optimized for the maximum $\mathrm{k}_{\text {eff. }}$

- The scrap was assumed to fill the entire scrap basket volume in the $\mathrm{MCO}$, including the small volume above the top scrap basket below the filter guard plate.

- The scrap baskets did not include copper dividers.

- Scrap was excluded from the inner central pipe region of the scrap basket.

- Mark 1A scrap was enriched to $1.25 \mathrm{wt} \% \mathrm{U}^{235}$, no credit for burn-up was taken.

- The fuel baskets were modeled as being filled.

\subsection{Methods of Calculation}

MCNP4B was used for all calculations. For completeness, the calculations were performed by modeling two misloaded scrap baskets with three misloaded fuel baskets, a misloaded scrap basket in either the top and bottom tier of the MCO with four misloaded fuel baskets, and five misloaded fuel baskets. Because of the limited volume in the $\mathrm{MCO}$, a full scrap basket load of $980 \mathrm{~kg}$ will not fit in the scrap basket at optimal conditions. Therefore, the scrap was modeled at optimum conditions, filling the scrap basket volume, for the maximum $\mathrm{k}_{\mathrm{eff}}$. This mass is slightly less than the maximum loading for a Mark IV scrap basket. Partially loaded fuel baskets were not considered. The calculated $k_{\text {eff }}$ was corrected for bias and uncertainties using the method described in HNF-SD-SNF-CSER-005, Revision 4. 


\subsection{Findings and Conclusions}

Table 1 lists the results.

Table 1: Results

\begin{tabular}{|l|c|c|c|c|}
\hline \multicolumn{1}{|c|}{ Description } & Case & $\mathrm{k}_{\text {calc }}$ & $\sigma_{\mathrm{c}}$ & $\mathrm{k}_{\text {eff }}$ \\
\hline $\begin{array}{l}\text { Two misloaded scrap baskets and three } \\
\text { misloaded fuel baskets }\end{array}$ & $\mathrm{mk4r.1 \textrm {k }}$ & 0.97561 & 0.00077 & 0.98666 \\
\hline $\begin{array}{l}\text { Mark 1A scrap basket in the bottom tier with } \\
\text { four misloaded fuel baskets }\end{array}$ & $\mathrm{mk4r.1 \textrm {g }}$ & 0.97560 & 0.00075 & 0.98664 \\
\hline $\begin{array}{l}\text { Mark 1A scrap basket in the top tier with } \\
\text { four misloaded fuel baskets }\end{array}$ & $\mathrm{mk4r.1 \textrm {h }}$ & 0.97149 & 0.00086 & 0.98256 \\
\hline Five misloaded fuel baskets & $\mathrm{mk4r.1 \textrm {n }}$ & 0.86237 & 0.00082 & 0.87343 \\
\hline
\end{tabular}

$\left.k_{\text {eff }}=k_{\text {calc }}+0.0004+\left([0.01]^{2}+[1.645]^{2} \sigma_{c}^{2}+0.002083^{2}\right]\right)^{1 / 2}$

The results show that a misloaded MCO containing scrap baskets, when flooded, will exceed a $\mathrm{k}_{\text {eff }}$ of 0.95 but will still be subcritical. A completely misloaded MCO containing only fuel baskets will not exceed the 0.95 limit. ANSI/ANS STD 8.1, DOE Order 5480.24, and the HNF-PRO's require application of the double contingency principle to ensure a criticality accident is not possible due to a single change in process conditions. The complete misloading of an MCO would require multiple violations of administrative controls. An inventory listing the contents of each storage canister exists for both basins. This inventory is used to develop the fuel retrieval sequence to minimize handling Mark 1A and Mark IV simultaneously. Because the fuels are stored in different types of canisters, operators can visually distinguish what type of fuel they are handling. This represents two controls over the loading of baskets that would have to be violated repeatedly to completely misload an MCO. 


\title{
6.0 MCNP Input Files
}

imk4r.1k

Basic model for MCO loaded with 2 scrap baskets and 3 fuel baskets.

c All fuel baskets loaded with 20.9 in mkla assemblies. Both scrap baskets

c Contain mkla scrap.

c Scrap baskets contain the central pipe.

c Revised $1.25^{\prime \prime}$ basket base plates for all baskets

c Fuel basket containing intact fuel assemblies

$\begin{array}{lllllllllll}2 & 0 & -17 & 18 & -19 & 20 & -21 & 22 & \mathrm{u}=8 & \text { lat }=2\end{array}$ fill $=-5: 5 \quad-5: 5 \quad 0: 0$

$\begin{array}{lllllllllllll}2 & 2 & 2 & 2 & 2 & 2 & 2 & 2 & 2 & 2 & 2 & \$ & 1\end{array}$

$\begin{array}{lllllllllllll}2 & 2 & 2 & 2 & 2 & 2 & 3 & 3 & 3 & 2 & 2 & \$ & 2\end{array}$

$\begin{array}{lllllllllllll}2 & 2 & 2 & 2 & 3 & 3 & 3 & 3 & 3 & 3 & 2 & \$ & 3\end{array}$

$\begin{array}{lllllllllllll}2 & 2 & 2 & 3 & 3 & 3 & 3 & 3 & 3 & 3 & 2 & \$ & 4\end{array}$

$\begin{array}{lllllllllllll}2 & 2 & 3 & 3 & 3 & 3 & 3 & 3 & 3 & 3 & 2 & \$ & 5\end{array}$

$\begin{array}{lllllllllllllll}2 & 2 & 3 & 3 & 3 & 2 & 3 & 3 & 3 & 2 & 2 & \$ & 6 & \text { level } 1\end{array}$

$\begin{array}{lllllllllllll}2 & 3 & 3 & 3 & 3 & 3 & 3 & 3 & 3 & 2 & 2 & \$ 7 & 7\end{array}$

$\begin{array}{lllllllllllll}2 & 3 & 3 & 3 & 3 & 3 & 3 & 3 & 2 & 2 & 2 & \$ 8\end{array}$

$\begin{array}{llllllllllll}2 & 3 & 3 & 3 & 3 & 3 & 3 & 2 & 2 & 2 & 2 & \$ 9\end{array}$

$\begin{array}{lllllllllllll}2 & 2 & 3 & 3 & 3 & 2 & 2 & 2 & 2 & 2 & 2 & \$ 10\end{array}$

$\begin{array}{llllllllllll}2 & 2 & 2 & 2 & 2 & 2 & 2 & 2 & 2 & 2 & 2 & \$ 11\end{array}$

$\begin{array}{lllllllllllll}\text { c } & 1 & 2 & 3 & 4 & 5 & 6 & 7 & 8 & 9 & 10 & 11\end{array}$

c Mark 4 scrap optimized lattice

$50 \quad-11 \backslash 2-1314-1516$ fill=4 $u=1$ lat $=2 \$$ scrap hex

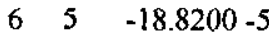

$\begin{array}{lll}7 & 10 & -6.490005-6\end{array}$

$8236-1.000006$

$\mathrm{u}=4 \quad \$$ scrap pin

$\mathrm{u}=4 \quad \$$ scrap clad

$\mathrm{u}=4 \quad \$$ scrap cell

c Scrap basket

\begin{tabular}{|c|c|c|c|c|c|}
\hline 0 & \multicolumn{2}{|c|}{ 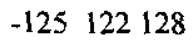 } & & fill $=1 \quad u=9$ & $\$$ scrap in basket \\
\hline 20 & -8.03 & -128 & $127 \quad 122$ & $\mathbf{u}=9$ & $9 \$$ \$ central pipe \\
\hline 236 & -1.00 & -127 & -122 & $u=9$ & $\$$ pipe interior \\
\hline 20 & -8.03 & -122 & $127-125$ & $\mathrm{u}=\mathrm{c}$ & $\$$ basket base \\
\hline 20 & -8.03 & 125 & -126 & $u=9$ & $\$$ basket side \\
\hline 236 & -1.00 & 126 & & $u=9$ & \$ outside basket \\
\hline 236 & -1.00 & -127 & 122 & $\mathrm{u}=9$ & $\$$ inside centra \\
\hline
\end{tabular}

c fictitious water cell for fuel basket lattice

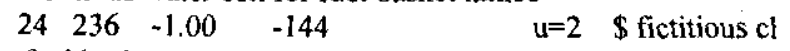

c fuel basket

$\begin{array}{lllllll}25 & 20 & -8.03 & -128 & 127 & 122 & -123\end{array}$

$\begin{array}{lllll}26 & 236 & -1.00 & -127 & -123\end{array}$

$\begin{array}{llllll}27 & 20 & -8.03 & -122 & 127 & -130\end{array}$

$\begin{array}{llllll}28 & 20 & -8.03 & -128 & 127 & 123\end{array}$

$\begin{array}{lllll}29 & 236 & -1.00 & -127 & 123\end{array}$

$\begin{array}{llllll}31 & 236 & -1.00 & -129 & 128 & 123\end{array}$

$32 \quad 20-8.03000129122-130$

$33 \quad 0 \quad-129122128-123$

$\begin{array}{llll}34 & 236 & -1.00 \quad 130\end{array}$

c fuel and scrap basket stack

$51 \quad 0 \quad-149 \quad$ fill $=19 \quad \mathrm{u}=11 \quad \$ \mathrm{mk}$ la scrap basket

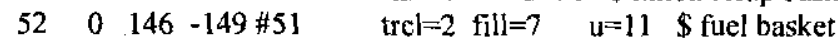

$53 \quad 0 \quad 146-149 \# 52 \quad$ trcl $=3$ fill $=7 \quad \mathrm{u}=11 \quad \$$ fuel basket

$54 \quad 0 \quad 146-149 \# 53 \quad$ trcl $=4$ fill $=7 \quad u=11 \quad \$$ fuel basket

$55 \quad 0 \quad 146-149 \# 54 \quad$ trcl=5 fill=19 u=11 $\$$ mkl a scrap basket

$59 \quad 0 \quad 152 \# 55 \quad$ fill $=20 \quad u=11 \quad \$$ mk la scrap above top basket

c MCO and baskets

$\begin{array}{llr}60 & 0 & (-160146-150)\end{array}$

$61 \quad 20-8.03 \quad 160-161146$

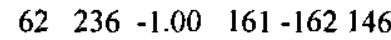

\author{
fill=11 $u=12 \quad \$$ interior \\ $u=12 \quad \$$ MCO shell \\ $u=12$ \$ cask annulus
}




\begin{tabular}{|c|c|c|c|c|}
\hline 63 & $20-$ & -8.03 & \multicolumn{2}{|c|}{146} \\
\hline 64 & $20-$ & -8.03 & \multicolumn{2}{|c|}{-146} \\
\hline 65 & $20-$ & $-8.03-1$ & \multicolumn{2}{|c|}{165} \\
\hline 66 & $20-$ & $-8.03-1$ & \multicolumn{2}{|c|}{$150-151$} \\
\hline 67 & 236 & .1 .00 & \multicolumn{2}{|c|}{$151-165$} \\
\hline 68 & 0 & 164 & \multicolumn{2}{|c|}{$63-166$} \\
\hline \multicolumn{5}{|c|}{ c Mkla intact fuel structure } \\
\hline 115 & 236 & -1.00 & \multicolumn{2}{|c|}{50} \\
\hline 116 & 10 & -6.49 & -50 & 49 \\
\hline 117 & 4 & -18.82 & -49 & 48 \\
\hline 118 & 10 & -6.49 & -48 & 47 \\
\hline 119 & 236 & -1.00 & -47 & 46 \\
\hline 120 & 10 & -6.49 & -46 & 45 \\
\hline 121 & 5 & -18.82 & -45 & 44 \\
\hline 122 & 10 & -6.49 & -44 & 43 \\
\hline 123 & 236 & -1.00 & -43 & \\
\hline
\end{tabular}

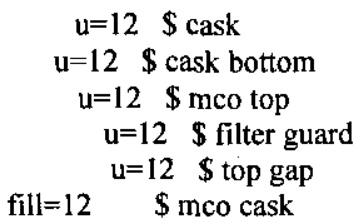

c Mark la scrap optimized lattice
$\begin{array}{llllllll}125 & 0 & -181 & 182 & -183 & 184 & -185 & 186\end{array}$
$\begin{array}{lll}126 & 4 & -18.8200-175\end{array}$

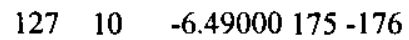
$\begin{array}{lll}128 & 236 & -1.00000176\end{array}$

c mkla Scrap basket

\begin{tabular}{|c|c|c|c|c|c|}
\hline 129 & 0 & -195 & 122198 & fill $=20 u=19$ & \$ scrap in basket \\
\hline 130 & 236 & -1.00 & -197 & $u=19$ & $\$$ pipe interior \\
\hline 131 & 20 & -8.03 & -122 & $197-195$ & $\$$ basket base \\
\hline 132 & 0 & & 195 & $-196 \quad$ fill $=20 \quad u=19$ & $\$$ basket side \\
\hline 133 & 0 & & 196 & fill $=20 u=19 s$ & $\$$ outside basket \\
\hline 134 & 20 & -8.03 & -198 & 197122 & 9 \$ central pipe \\
\hline 135 & 236 & -1.00 & $: 197$ & 122 & $\$$ inside central pipe \\
\hline 145 & 2 & 0.065 & $20 \cdot 164$ & $141-144$ & $\$$ basin floor \\
\hline 146 & 236 & -1.000 & $-144-1$ & 143166 & \$ water above MCO \\
\hline 147 & 236 & -1.000 & -1661 & $63164-144$ & $\$$ surrounding water \\
\hline 149 & 0 & $141: 14$ & $4: 143$ & & utside world \\
\hline
\end{tabular}

\begin{tabular}{|c|c|c|c|c|c|}
\hline 5 & $\mathrm{cz}$ & 0.8000000 & & & \\
\hline 6 & cz & 0.8572000 & & & \\
\hline 11 & $\mathrm{p}$ & 0.8660254 & -0.5000000 & 0.0000000 & 1.3992000 \\
\hline 12 & $\mathrm{p}$ & 0.8660254 & -0.5000000 & 0.0000000 & -1.3992000 \\
\hline 13 & $\mathrm{p}$ & 0.8660254 & 0.5000000 & 0.0000000 & 1.3992000 \\
\hline 14 & $\mathrm{p}$ & 0.8660254 & 0.5000000 & 0.0000000 & -1.3992000 \\
\hline 15 & py & 1.3992000 & & & \\
\hline 16 & py & -1.3992000 & & & \\
\hline 17 & p & 0.86602540 & $\begin{array}{ll}-0.5 & 0.0\end{array}$ & 3.4925 & \\
\hline 18 & p & 0.86602540 & $-0.5 \quad 0.0$ & -3.4925 & \\
\hline 19 & $\mathrm{p}$ & 0.86602540 & $0.5 \quad 0.0$ & 3.4925 & \\
\hline 20 & $\mathrm{p}$ & 0.86602540 & $0.5 \quad 0.0$ & -3.4925 & \\
\hline 21 & py & \multicolumn{4}{|c|}{3.4925} \\
\hline 22 & py & \multicolumn{4}{|c|}{-3.4925} \\
\hline 43 & $\mathrm{cz}$ & 0.5588 & \multicolumn{3}{|l|}{$\$ \mathrm{H} 2 \mathrm{O}$} \\
\hline 44 & $c z$ & 0.6223 & \multicolumn{3}{|l|}{$\$ \mathrm{Zr}-2 \mathrm{clad}$} \\
\hline 45 & $\mathrm{cz}$ & 1.4808 & \multicolumn{3}{|l|}{$\$$ fuel } \\
\hline 46 & cz & 1.5824 & \multicolumn{3}{|l|}{$\$ \mathrm{Zr}-2 \mathrm{clad}$} \\
\hline 47 & $\mathrm{cz}$ & 2.2441 & \multicolumn{3}{|l|}{$\$ \mathrm{H} 2 \mathrm{O}$} \\
\hline 48 & $c z$ & 2.3076 & \multicolumn{3}{|l|}{$\$ \mathrm{Zr}-2$ clad } \\
\hline 49 & cz & 2.9896 & \multicolumn{3}{|l|}{$\$$ fuel } \\
\hline 50 & $\mathrm{cz}$ & 3.0531 & \multicolumn{3}{|l|}{$\$ \mathrm{Zr}-2 \mathrm{clad}$} \\
\hline 122 & $\mathrm{pz}$ & 0.0000000 & & & \\
\hline 123 & $\mathrm{pz}$ & 53.086000 & & & \\
\hline \multicolumn{6}{|c|}{ c scrap basket side } \\
\hline 125 & $\mathrm{cz}$ & 28.79725000 & & & \\
\hline 126 & $c z$ & 29.08751997 & & & \\
\hline
\end{tabular}




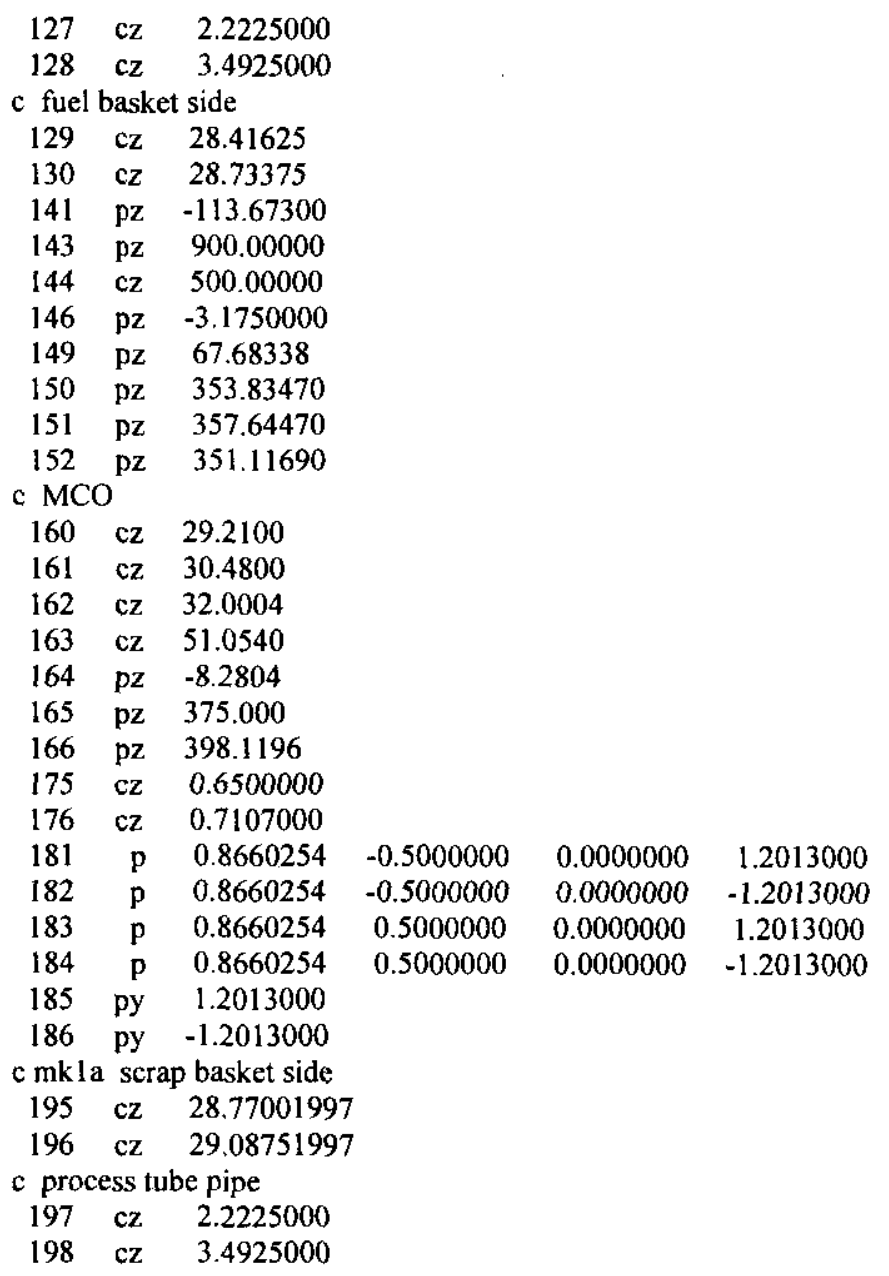

tr2 $\quad 0.00 \quad 0.00 \quad 70.858380$

tr3 $0.00 \quad 0.00 \quad 141.71676$

$\begin{array}{lllll}\text { tr4 } & 0.00 & 0.00 & 212.57514\end{array}$

tr5 $0.00 \quad 0.00 \quad 283.43352$

mode $\mathrm{n}$

$\begin{array}{llllll}\text { m4 } & 92235.50 & -0.01250 & 92238.50 & -0.98750 & \text {. \$ outer fuel } \\ \text { m5 } & 92235.50 & -0.00947 & 92238.50 & -0.99053 & \text { \$ inner fuel }\end{array}$

$\mathrm{m} 2361001.50 \mathrm{c} 0.6667008016 .50 \mathrm{c} 0.333300$

$\mathrm{m} 10 \quad 40000.50 \mathrm{c}-1.000000$

$\mathrm{m} 2026000.55 \mathrm{c} 67.97000124000 .50 \mathrm{c} 20.00000028000 .50 \mathrm{c} 10.000000$

$25055.50 \mathrm{c} 2.0000006000 .50 \mathrm{c} 0.030000$

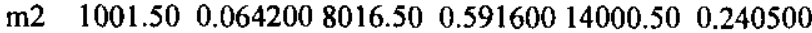

$20000.50 \quad 0.07380026000 .55 \quad 0.029900$

imp:n $\quad 1.00000 \quad 59 \mathrm{r} \quad 0.00000 \$ 5,139$

$\mathrm{mt} 236$ lwtr.01t

$\mathrm{mt} 2$ lwtr.01t

print 406080100110126

prdmp j 300

kcode 20001.20300

ksrc $9.70 .20 .-9.70 .085 .09 .7-9.7150 .0$

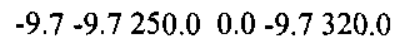

imk4r.1g

Basic model for MCO loaded with 1 scrap basket and 4 fuel baskets.

c All fuel baskets loaded with 20.9 in mk la assemblies. Bottom scrap basket 
c contains mk la scrap.

c Scrap baskets contain the central pipe.

c Revised $1.25^{\prime \prime}$ basket base plates for all baskets.

c fuel basket containing intact fuel assemblies

$\begin{array}{llllllllll}2 & 0 & -17 & 18 & -19 & 20 & -21 & 22 & u=8 & l a t=2\end{array}$

$$
\text { fill }=-5: 5 \quad-5: 5 \quad 0: 0
$$

$\begin{array}{lllllllllllll}2 & 2 & 2 & 2 & 2 & 2 & 2 & 2 & 2 & 2 & 2 & \$ 1\end{array}$

$\begin{array}{lllllllllllll}2 & 2 & 2 & 2 & 2 & 2 & 3 & 3 & 3 & 2 & 2 & \$ & 2\end{array}$

$\begin{array}{lllllllllllll}2 & 2 & 2 & 2 & 3 & 3 & 3 & 3 & 3 & 3 & 2 & \$ 3\end{array}$

$\begin{array}{lllllllllllll}2 & 2 & 2 & 3 & 3 & 3 & 3 & 3 & 3 & 3 & 2 & \$ & 4\end{array}$

$\begin{array}{lllllllllllll}2 & 2 & 3 & 3 & 3 & 3 & 3 & 3 & 3 & 3 & 2 & \$ 5\end{array}$

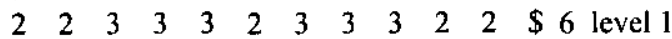

$\begin{array}{lllllllllllll}2 & 3 & 3 & 3 & 3 & 3 & 3 & 3 & 3 & 2 & 2 & \$ 7\end{array}$

$\begin{array}{lllllllllllll}2 & 3 & 3 & 3 & 3 & 3 & 3 & 3 & 2 & 2 & 2 & \$ 8\end{array}$

$\begin{array}{lllllllllllll}2 & 3 & 3 & 3 & 3 & 3 & 3 & 2 & 2 & 2 & 2 & \$ 9\end{array}$

$\begin{array}{llllllllllll}2 & 2 & 3 & 3 & 3 & 2 & 2 & 2 & 2 & 2 & 2 & \$ 10\end{array}$

$\begin{array}{llllllllllll}2 & 2 & 2 & 2 & 2 & 2 & 2 & 2 & 2 & 2 & 2 & \$ 11\end{array}$

$\begin{array}{llllllllllll}\text { c } & 1 & 2 & 3 & 4 & 5 & 6 & 7 & 8 & 9 & 10 & 11\end{array}$

c Mark 4 scrap optimized lattice

$$
\begin{aligned}
& 5 \quad 0 \quad-11 \quad 12-13 \quad 14-15 \quad 16 \text { fill=4 } u=1 \text { lat=2 } \$ \text { scrap hex } \\
& 6 \quad 5 \quad-18.8200-5 \quad \mathrm{u}=4 \quad \text { \$ scrap pin } \\
& 7 \quad 10 \quad-6.490005-6 \quad u=4 \quad \text { \$ scrap clad } \\
& 8236-1.000006 \quad u=4 \quad \$ \text { scrap cell }
\end{aligned}
$$

c Scrap basket

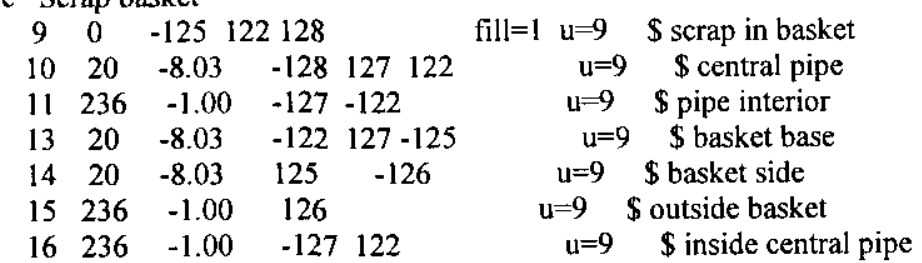

c fictitious water cell for fuel basket lattice

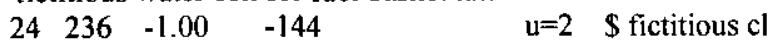

c fuel basket

$$
\begin{aligned}
& \begin{array}{lllllllll}
25 & 20 & +8.03 & -128 & 127 & 122 & -123 \quad \mathrm{u}=7 \quad \$ \text { tube wall }
\end{array} \\
& \begin{array}{lllllll}
26 & 236 & -1.00 & -127 & -123 & u=7 & \$ \text { central tube }
\end{array} \\
& \begin{array}{lllllll}
27 & 20 & -8.03 & -122 & 127 & -130 & \mathrm{u}=7
\end{array} \text { \$ bottom plate } \\
& \begin{array}{llllllll}
28 & 20 & -8.03 & -128 & 127 & 123 & \mathrm{u}=7 & \$ \text { tube wall }
\end{array} \\
& \begin{array}{lllllll}
29 & 236 & -1.00 & -127 & 123 & u=7 & \$ \text { central tube }
\end{array} \\
& \begin{array}{lllllll}
31 & 236 & -1.00 & -129 & 128 & 123 & \mathrm{u}=7 \quad \text { \$ above fuel }
\end{array} \\
& 32 \quad 20-8.03000129122-130 \quad \mathrm{u}=7 \quad \$ \text { fuel basket side } \\
& 33 \quad 0-129122128-123 \quad \text { fill }=8 \mathrm{u}=7 \quad \$ \text { fuel in basket } \\
& \begin{array}{llll}
34 & 236 & -1.00 & 130
\end{array}
\end{aligned}
$$

c fuel and scrap basket stack

$$
\begin{aligned}
& 51 \quad 0 \quad-149 \quad \text { fill=19 } \quad \mathrm{u}=11 \quad \$ \mathrm{mk} \text { la scrap basket } \\
& 52 \quad 0 \quad 146-149 \# 51 \quad \text { trcl }=2 \text { fill }=7 \quad \mathrm{u}=11 \quad \text { \& fuel basket } \\
& \begin{array}{llllll}
53 & 0 & 146 & -149 \# 52 \quad \text { trcl }=3 \text { fill }=7 \quad \mathrm{u}=11 \quad \text { \$ fuel basket }
\end{array} \\
& 54 \quad 0 \quad 146-149 \# 53 \quad \text { trcl=4 fill=7 } \quad \mathrm{u}=11 \quad \text { \$ fuel basket } \\
& 55 \quad 0 \quad 146 \quad-149 \quad \# 54 \quad \text { trcl=5 fill=7 } \quad \mathrm{u}=11 \quad \text { \& fuel basket } \\
& \begin{array}{lllll}
59 & 236 & -1.000 \quad 152 \quad \# 55 \quad \mathrm{u}=11 \quad \text { \$ above top basket }
\end{array}
\end{aligned}
$$

c MCO and baskets

$$
\begin{aligned}
& 60 \quad 0 \quad(-160146-150) \\
& \begin{array}{llllll}
61 & 20 & -8.03 & 160 & -161 & 146
\end{array}
\end{aligned}
$$

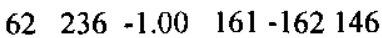

$$
\begin{aligned}
& \begin{array}{lllll}
63 & 20 & -8.03 & 162 & 146
\end{array} \\
& \begin{array}{llll}
64 & 20 & -8.03 & -146
\end{array} \\
& \begin{array}{llllll}
65 & 20 & -8.03 & -160 & 165
\end{array} \\
& \begin{array}{lllllll}
66 & 20 & -8.03 & -160 & 150 & -151
\end{array} \\
& \begin{array}{llllll}
67 & 236 & -1.00 & -160 & 151 & -165
\end{array} \\
& \begin{array}{lllll}
68 & 0 & 164 & -163 & -166
\end{array} \\
& \text { c Mkla intact fuel structure } \\
& \begin{array}{llll}
115 & 236 & -1.00 & 50
\end{array}
\end{aligned}
$$

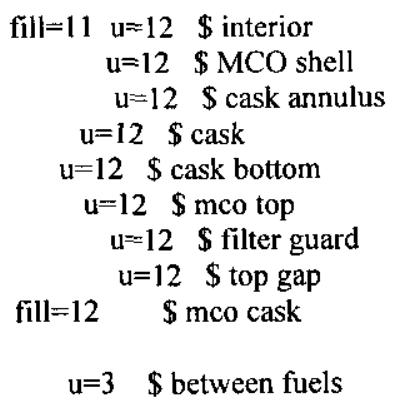

$u=3 \quad \$$ between fuels 


$\begin{array}{ccccc}116 & 10 & -6.49 & -50 & 49 \\ 117 & 4 & -18.82 & -49 & 48 \\ 118 & 10 & -6.49 & -48 & 47 \\ 119 & 236 & -1.00 & -47 & 46 \\ 120 & 10 & -6.49 & -46 & 45 \\ 121 & 5 & -18.82 & -45 & 44 \\ 122 & 10 & -6.49 & -44 & 43 \\ 123 & 236 & -1.00 & -43 & \end{array}$

c Mark la scrap optimized lattice

$$
\begin{array}{cl}
u=3 & \$ \mathrm{Zr}-2 \text { clad } \\
\mathrm{u}=3 & \$ 1.250 \% \mathrm{U}-235 \\
\mathrm{u}=3 & \$ \mathrm{Zr}-2 \text { clad } \\
\mathrm{u}=3 & \$ \mathrm{H} 2 \mathrm{O} \\
\mathrm{u}=3 & \$ \mathrm{Zr}-2 \text { clad } \\
\mathrm{u}=3 & \$ 0.947 \% \mathrm{U}-235 \\
\mathrm{u}=3 & \$ \mathrm{Zr}-2 \text { clad } \\
\mathrm{u}=3 & \$ \mathrm{H} 2 \mathrm{O}
\end{array}
$$
$1250 \quad-181 \quad 182-183184-185186$ fill=14 u=20 lat=2 \$ scrap hex
$\begin{array}{lll}126 & 4 & -18.8200-175\end{array}$
$127 \quad 10 \quad-6.49000175-176$
$128 \quad 236 \quad-1.00000176$
c mkla Scrap basket

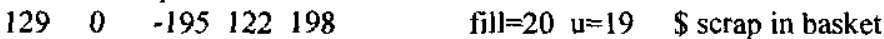
$\begin{array}{lllllll}130 & 236 & -1.00 & -197 & -122 & \mathrm{u}=19 & \text { \$ pipe interior }\end{array}$
$\begin{array}{lllllll}131 & 20 & -8.03 & -122 & 197 & -195 \quad \mathrm{u}=19 & \text { \$ basket base }\end{array}$
$1320 \quad 195 \quad-196 \quad$ fill $=20 u=19 \quad \$$ basket side
$1330 \quad 196 \quad$ fill $=20 u=19 \quad$ S outside basket
$\begin{array}{llllllll}134 & 20 & -8.03 & -198 & 197 & 122 & \mathrm{u}=19 & \text { \$ central pipe }\end{array}$
$\begin{array}{llllll}135 & 236 & -1.00 & -197 & 122 & u=19\end{array}$ \$ inside central pipe
$14520.06520-164141-144$

\begin{tabular}{|c|c|c|c|c|c|}
\hline 5 & $c z$ & 0.8000000 & & & \\
\hline 6 & $\mathrm{cz}$ & 0.8572000 & & & \\
\hline 11 & $\mathrm{p}$ & 0.8660254 & -0.5000000 & 0.0000000 & 1.3992000 \\
\hline 12 & p & 0.8660254 & -0.5000000 & 0.0000000 & -1.3992000 \\
\hline 13 & $\mathrm{p}$ & 0.8660254 & 0.5000000 & 0.0000000 & 1.3992000 \\
\hline 14 & $\mathrm{p}$ & 0.8660254 & 0.5000000 & 0.0000000 & -1.3992000 \\
\hline 15 & py & 1.3992000 & & & \\
\hline 16 & py & -1.3992000 & & & \\
\hline 17 & p & 0.86602540 & $-0.5 \quad 0.0$ & 3.4925 & \\
\hline 18 & $\mathrm{p}$ & 0.86602540 & $\begin{array}{ll}-0.5 & 0.0\end{array}$ & -3.4925 & \\
\hline 19 & $\mathrm{p}$ & 0.86602540 & $0.5 \quad 0.0$ & 3.4925 & \\
\hline 20 & $\mathrm{p}$ & 0.86602540 & $0.5 \quad 0.0$ & -3.4925 & \\
\hline 21 & py & & 3.4925 & & \\
\hline 22 & py & & -3.4925 & & \\
\hline 43 & $\mathrm{cz}$ & 0.5588 & $\$ \mathrm{H} 2 \mathrm{O}$ & & \\
\hline 44 & $\mathrm{cz}$ & 0.6223 & $\$ \mathrm{Zr}-2 \mathrm{clad}$ & & \\
\hline 45 & $\mathrm{cz}$ & 1.4808 & $\$$ fuel & & \\
\hline 46 & $\mathrm{cz}$ & 1.5824 & $\$ \mathrm{Zr}-2$ clad & & \\
\hline 47 & $\mathrm{cz}$ & 2.2441 & $\$ \mathrm{H} 2 \mathrm{O}$ & & \\
\hline 48 & $\mathrm{cz}$ & 2.3076 & $\$ \mathrm{Zr}-2 \mathrm{clad}$ & & \\
\hline 49 & $c z$ & 2.9896 & $\$$ fuel & & \\
\hline 50 & $\mathrm{cz}$ & 3.0531 & $\$ \mathrm{Zr}-2 \mathrm{clad}$ & & \\
\hline 122 & $\mathrm{pz}$ & 0.0000000 & & & \\
\hline 123 & $\mathrm{pz}$ & 53.086000 & & & \\
\hline \multicolumn{6}{|c|}{ scrap basket side } \\
\hline 125 & $c z$ & 28.7972500 & & & \\
\hline 126 & $\mathrm{cz}$ & 29.0875199 & & & \\
\hline \multicolumn{6}{|c|}{ process tube pipe } \\
\hline 127 & $\mathrm{cz}$ & 2.2225000 & & & \\
\hline 128 & $c z$ & 3.4925000 & & & \\
\hline \multicolumn{6}{|c|}{ fuel basket side } \\
\hline 129 & $c z$ & 28.41625 & & & \\
\hline 130 & $c z$ & 28.73375 & & & \\
\hline 141 & $\mathrm{p} z$ & -113.67300 & & & \\
\hline 143 & $\mathrm{pz}$ & 900.00000 & & & \\
\hline 144 & $\mathrm{cz}$ & 500.00000 & & & \\
\hline
\end{tabular}
$\begin{array}{llllll}146 & 236 & -1.000 & -144 & -143 & 166\end{array}$
$147 \quad 236-1.000-166 \quad 163164-144$
$149 \quad 0 \quad-141: 144: 143$
$\$$ basin floor
$\$$ water above MCO
$\$$ surrounding water $\$$ outside world




\begin{tabular}{|c|c|c|c|c|c|}
\hline 146 & $\mathrm{pz}$ & -3.1750000 & & & \\
\hline 149 & $\mathrm{pz}$ & 67.68338 & & & \\
\hline 150 & $\mathrm{pz}$ & 353.83470 & & & \\
\hline 151 & $\mathrm{pz}$ & 357.64470 & & & \\
\hline 152 & $\mathrm{pz}$ & 351.11690 & & & \\
\hline \multicolumn{6}{|c|}{ c $\mathrm{MCO}$} \\
\hline 160 & $\mathrm{cz}$ & 29.2100 & & & \\
\hline 161 & $\mathrm{cz}$ & 30.4800 & & & \\
\hline 162 & $\mathrm{cz}$ & 32.0004 & & & \\
\hline 163 & $\mathrm{cz}$ & 51.0540 & & & \\
\hline 164 & $\mathrm{pz}$ & -8.2804 & & & \\
\hline 165 & $\mathrm{pz}$ & 375.000 & & & \\
\hline 166 & $\mathrm{pz}$ & 398.1196 & & & \\
\hline 175 & cz & 0.6500000 & & & \\
\hline 176 & $\mathrm{cz}$ & 0.7107000 & & & \\
\hline 181 & $\mathrm{p}$ & 0.8660254 & -0.5000000 & 0.0000000 & 1.2013000 \\
\hline 182 & $\mathrm{p}$ & 0.8660254 & -0.5000000 & 0.0000000 & -1.2013000 \\
\hline 183 & p & 0.8660254 & 0.5000000 & 0.0000000 & 1.2013000 \\
\hline 184 & $\mathrm{p}$ & 0.8660254 & 0.5000000 & 0.0000000 & -1.2013000 \\
\hline 185 & py & 1.2013000 & & & \\
\hline 186 & py & -1.2013000 & & & \\
\hline \multicolumn{6}{|c|}{ c mk la scrap basket side } \\
\hline 195 & $\mathrm{cz}$ & 28.7700199 & & & \\
\hline 196 & $\mathrm{cz}$ & 29.08751997 & & & \\
\hline \multicolumn{6}{|c|}{ c process tube pipe } \\
\hline 197 & $\mathrm{cz}$ & 2.2225000 & & & \\
\hline 198 & $\mathrm{cz}$ & 3.4925000 & & & \\
\hline
\end{tabular}

tr2 $\quad 0.00 \quad 0.00 \quad 70.858380$

tr3 $0.00 \quad 0.00 \quad 141.71676$

$\begin{array}{lllll}\text { tr4 } & 0.00 & 0.00 & 212.57514\end{array}$

tr5 $\quad 0.00 \quad 0.00 \quad 283.43352$

mode $\mathrm{n}$

m4 $\quad 92235.50 \quad-0.01250 \quad 92238.50 \quad-0.98750 \quad$ \$ outer fuel

m5 $\quad 92235.50 \quad-0.00947 \quad 92238.50 \quad-0.99053 \quad \$$ inner fuel

$\mathrm{m} 2361001.50 \mathrm{c} 0.6667008016 .50 \mathrm{c} 0.333300$

$\mathrm{m} 10 \quad 40000.50 \mathrm{c}-1.000000$

$\mathrm{m} 2026000.55 \mathrm{c} 67.97000124000 .50 \mathrm{c} 20.00000028000 .50 \mathrm{c} 10.000000$ $25055.50 \mathrm{c} 2.0000006000 .50 \mathrm{c} 0.030000$

$\begin{array}{lllllll}\mathrm{m} 2 & 1001.50 & 0.064200 & 8016.50 & 0.591600 & 14000.50 & 0.240500\end{array}$

$20000.50 \quad 0.07380026000 .55 \quad 0.029900$

imp:n $\quad 1.00000 \quad 59 \mathrm{r} \quad 0.00000 \$ 5,139$

mt236 lwtr.0It

$\mathrm{mt} 2$ lwtr.01t

print 406080100110126

prdmp j 300

kcode 20001.20300

ksrc $9.70 .20 . \quad-9.70 .085 .09 .7-9.7150 .0$

$-9.7-9.7250 .0 \quad 0.0-9.7320 .0$

imk4r.1h

Basic model for MCO loaded with 1 scrap basket and 4 fuel baskets.

c All fuel baskets loaded with 20.9 in mk la assemblies. Top scrap basket

c contains mkla scrap.

c Scrap baskets contain the central pipe.

c Revised 1.25" basket base plates for all baskets.

c fuel basket containing intact fuel assemblies

$\begin{array}{llllllllll}2 & 0 & -17 & 18 & -19 & 20 & -21 & 22 & u=8 & \text { lat=2 }\end{array}$ fill $=-5: 5 \quad-5: 5 \quad 0: 0$

$\begin{array}{lllllllllllll}2 & 2 & 2 & 2 & 2 & 2 & 2 & 2 & 2 & 2 & 2 & \$ 1\end{array}$

$\begin{array}{lllllllllllll}2 & 2 & 2 & 2 & 2 & 2 & 3 & 3 & 3 & 2 & 2 & \$ 2\end{array}$ 


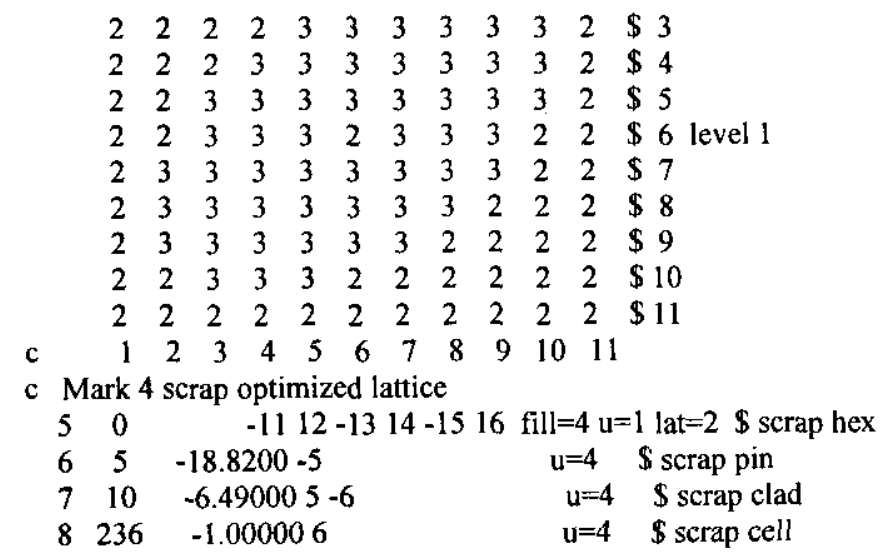

c Scrap basket

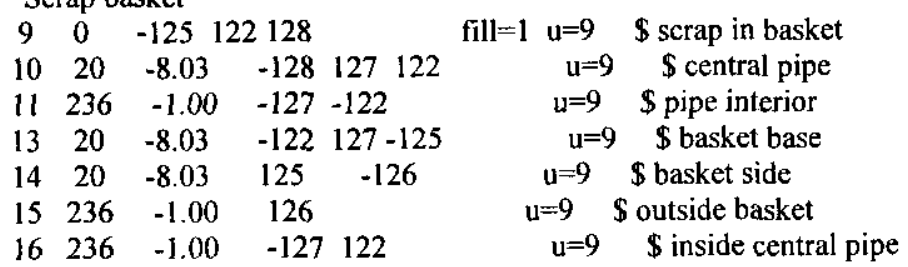

c fictitious water cell for fuel basket lattice
$\begin{array}{llll}24 & 236 & -1.00 & -144\end{array}$
$\mathrm{u}=2 \quad \$$ fictitious cl
c fuel basket

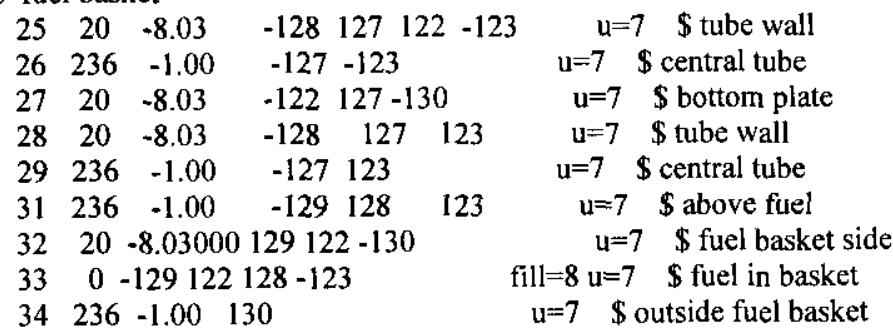

c fuel and scrap basket stack
510
$-149$
fill=7 $u=11 \quad \$$ fuel basket
$\begin{array}{lllll}52 & 0 & 146 & -149 & \# 51\end{array}$
$53 \quad 0 \quad 146-149$ \#52
$54 \quad 0 \quad 146-149$ \#53
trcl=2 fill=7 $\mathrm{u}=11 \quad \$$ fuel basket
trcl=3 fill=7 $u=11 \quad \$$ fuel basket
$\begin{array}{lllll}55 & 0 & 146 & -149 & \# 54\end{array}$
trcl=4 fill=7 $u=11 \quad \$$ fuel basket
590
$152 \# 55$
trcl= 5 fill=19 $u=11 \quad \$$ mkla scrap basket

c $\mathrm{MCO}$ and baskets
$60 \quad 0 \quad(-160146-150)$
$\begin{array}{llllll}61 & 20 & -8.03 & 160 & -161 & 146\end{array}$
$\begin{array}{lllll}62 & 236 & -1.00 & 161 & -162146\end{array}$
$\begin{array}{lllll}63 & 20 & -8.03 & 162 & 146\end{array}$
$\begin{array}{llll}64 & 20 & -8.03 & -146\end{array}$
$\begin{array}{llllll}65 & 20 & -8.03 & -160 & 165\end{array}$
$\begin{array}{llllll}66 & 20 & -8.03 & -160 & 150 & -151\end{array}$
$\begin{array}{llllll}67 & 236 & -1.00 & -160 & 151 & -165\end{array}$
$\begin{array}{llllll}68 & 0 & 164 & -163 & -166\end{array}$
c Mkla intact fuel structure

$\begin{array}{ccccc}115 & 236 & -1.00 & & 50 \\ 116 & 10 & -6.49 & -50 & 49 \\ 117 & 4 & -18.82 & -49 & 48 \\ 118 & 10 & -6.49 & -48 & 47 \\ 119 & 236 & -1.00 & -47 & 46 \\ 120 & 10 & -6.49 & -46 & 45 \\ 121 & 5 & -18.82 & -45 & 44 \\ 122 & 10 & -6.49 & -44 & 43 \\ 123 & 236 & -1.00 & -43 & \end{array}$
fill=11 $u=12 \quad \$$ interior $\mathrm{u}=12 \quad \$$ MCO shell
$\mathrm{u}=12 \quad \$$ cask annulus
$\mathrm{u}=12 \quad \$$ cask
$\mathrm{u}=12 \$$ cask bottom
$\mathrm{u}=12 \quad \$$ mco top
$u=12 \quad \$$ filter guard
$\mathrm{u}=12 \$$ top gap
fill $=12 \quad \$$ mco cask
$\mathrm{u}=3 \quad \$$ between fuels
$\mathrm{u}=3 \quad \$ \mathrm{Zr}-2$ clad
$\mathrm{u}=3 \quad \$ 1.250 \% \mathrm{U}-235$
$\mathrm{u}=3 \quad \$ \mathrm{Zr}-2 \mathrm{clad}$
$\mathrm{u}=3 \quad \$ \mathrm{H} 2 \mathrm{O}$
$\mathrm{u}=3 \quad \$ \mathrm{Zr}-2$ clad
$\mathrm{u}=3 \quad \$ 0.947 \% \mathrm{U}-235$
$\mathrm{u}=3 \quad \$ \mathrm{Zr}-2 \mathrm{clad}$
$\mathrm{u}=3 \quad \$ \mathrm{H} 2 \mathrm{O}$ 
c Mark 1a scrap optimized lattice

\begin{tabular}{|c|c|c|}
\hline 125 & 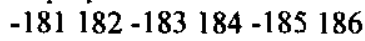 & fill=14 u=20 lat=2 $\$$ scrap hex \\
\hline 126 & $-18.8200-175$ & $\mathrm{u}=14 \quad \$$ scrap pin \\
\hline 127 & $-6.49000175-176$ & $u=14 \quad \$$ scrap clad \\
\hline 236 & -1.00000176 & $\$$ scrap cell \\
\hline
\end{tabular}
c mkla Scrap basket

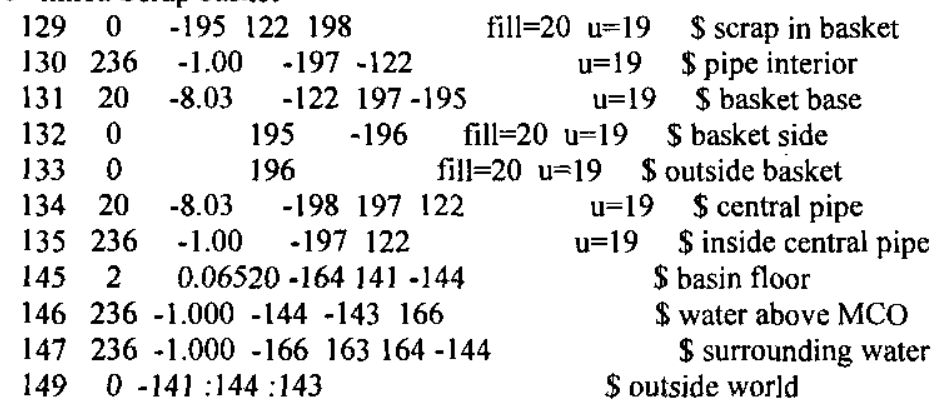

\begin{tabular}{|c|c|c|c|c|c|}
\hline 5 & $\mathrm{c} z$ & 0.8000000 & & & \\
\hline 6 & $\mathrm{cz}$ & 0.8572000 & & & \\
\hline 11 & $\mathrm{p}$ & 0.8660254 & -0.5000000 & 0.0000000 & 1.3992000 \\
\hline 12 & $\mathrm{p}$ & 0.8660254 & -0.5000000 & 0.0000000 & -1.3992000 \\
\hline 13 & $\mathrm{p}$ & 0.8660254 & 0.5000000 & 0.0000000 & 1.3992000 \\
\hline 14 & $\mathrm{p}$ & 0.8660254 & 0.5000000 & 0.0000000 & -1.3992000 \\
\hline 15 & py & 1.3992000 & & & \\
\hline 16 & py & -1.3992000 & & & \\
\hline 17 & $\mathrm{p}$ & 0.86602540 & $-0.5 \quad 0.0$ & 3.4925 & \\
\hline 18 & $\mathrm{p}$ & 0.86602540 & $-0.5 \quad 0.0$ & -3.4925 & \\
\hline 19 & p & 0.86602540 & 0.50 .0 & 3.4925 & \\
\hline 20 & $\mathrm{p}$ & 0.86602540 & $0.5 \quad 0.0$ & -3.4925 & \\
\hline 21 & py & & 3.4925 & & \\
\hline 22 & py & & -3.4925 & & \\
\hline 43 & $\mathrm{cz}$ & 0.5588 & $\$ \mathrm{H} 2 \mathrm{O}$ & & \\
\hline 44 & $c z$ & 0.6223 & $\$ \mathrm{Zr}-2 \mathrm{clad}$ & & \\
\hline 45 & $c z$ & 1.4808 & $\$$ fuel & & \\
\hline 46 & $\mathrm{cz}$ & 1.5824 & $\$ \mathrm{Zr}-2$ clad & & \\
\hline 47 & $\mathrm{cz}$ & 2.2441 & $\$ \mathrm{H} 2 \mathrm{O}$ & & \\
\hline 48 & $c z$ & 2.3076 & $\$ \mathrm{Zr}-2 \mathrm{clad}$ & & \\
\hline 49 & $c z$ & 2.9896 & $\$$ fuel & & \\
\hline 50 & $\mathrm{cz}$ & 3.0531 & $\$ \mathrm{Zr}-2$ clad & & \\
\hline 122 & $\mathrm{pz}$ & 0.0000000 & & & \\
\hline 123 & $\mathrm{pz}$ & 53.086000 & & & \\
\hline \multicolumn{6}{|c|}{ c scrap basket side } \\
\hline 125 & $\mathrm{cz}$ & 28.79725000 & & & \\
\hline 126 & $\mathrm{cz}$ & 29.0875199 & & & \\
\hline \multicolumn{6}{|c|}{ c process tube pipe } \\
\hline 127 & $\mathrm{cz}$ & 2.2225000 & & & \\
\hline 128 & $\mathrm{cz}$ & 3.4925000 & & & \\
\hline \multicolumn{6}{|c|}{ c fuel basket side } \\
\hline 129 & cz & 28.41625 & & & \\
\hline 130 & $\mathrm{cz}$ & 28.73375 & & & \\
\hline 141 & $\mathrm{pz}$ & -113.67300 & & & \\
\hline 143 & $\mathrm{pz}$ & 900.00000 & & & \\
\hline 144 & $\mathrm{cz}$ & 500.00000 & & & \\
\hline 146 & $p z$ & -3.1750000 & & & \\
\hline 149 & $\mathrm{pz}$ & 67.68338 & & & \\
\hline 150 & $\mathrm{pz}$ & 353.83470 & & & \\
\hline 151 & $\mathrm{pz}$ & 357.64470 & & & \\
\hline 152 & $\mathrm{pz}$ & 351.11690 & & & \\
\hline \multicolumn{6}{|c|}{ c $\mathrm{MCO}$} \\
\hline 160 & $\mathrm{cz}$ & 29.2100 & & & \\
\hline 161 & $\mathrm{cz}$ & 30.4800 & & & \\
\hline
\end{tabular}


SNF-4848 REV 0

$\begin{array}{llllll}162 & \mathrm{cz} & 32.0004 & & & \\ 163 & \mathrm{cz} & 51.0540 & & & \\ 164 & \mathrm{pz} & -8.2804 & & & \\ 165 & \mathrm{pz} & 375.000 & & & \\ 166 & \mathrm{pz} & 398.1196 & & & \\ 175 & \mathrm{cz} & 0.6500000 & & & \\ 176 & \mathrm{cz} & 0.7107000 & & & \\ 181 & \mathrm{p} & 0.8660254 & -0.5000000 & 0.0000000 & 1.2013000 \\ 182 & \mathrm{p} & 0.8660254 & -0.5000000 & 0.0000000 & -1.2013000 \\ 183 & \mathrm{p} & 0.8660254 & 0.5000000 & 0.0000000 & 1.2013000 \\ 184 & \mathrm{p} & 0.8660254 & 0.5000000 & 0.0000000 & -1.2013000 \\ 185 & \mathrm{py} & 1.2013000 & & & \\ 186 & \mathrm{py} & -1.2013000 & & & \\ \mathrm{c} \text { mk1a } & \mathrm{scrap} \text { basket side } & & & \\ 195 & \mathrm{cz} & 28.77001997 & & & \\ 196 & \mathrm{cz} & 29.08751997 & & & \\ \mathrm{c} \text { process tube } & \text { pipe } & & & \\ 197 & \mathrm{cz} & 2.2225000 & & & \\ 198 & \mathrm{cz} & 3.4925000 & & & \\ 7\end{array}$

tr2 $\quad 0.00 \quad 0.00 \quad 70.858380$

tr3 $\quad 0.00 \quad 0.00 \quad 141.71676$

tr4 $\quad 0.00 \quad 0.00 \quad 212.57514$

tr5 $\quad 0.00 \quad 0.00 \quad 283.43352$

mode $\mathrm{n}$

In4 $\quad 92235.50 \quad-0.01250 \quad 92238.50 \quad-0.98750 \quad$ \$ outer fuel m5 $\quad 92235.50 \quad-0.00947 \quad 92238.50 \quad-0.99053 \quad$ \$ inner fuel

$\mathrm{m} 2361001.50 \mathrm{c} 0.6667008016 .50 \mathrm{c} 0.333300$

$\mathrm{m} 10 \quad 40000.50 \mathrm{c}-1.000000$

$\mathrm{m} 2026000.55 \mathrm{c} 67.97000124000 .50 \mathrm{c} 20.00000028000 .50 \mathrm{c} 10.000000$

$25055.50 \mathrm{c} 2.0000006000 .50 \mathrm{c} 0.030000$

m2 $\quad \begin{array}{lllllll} & 1001.50 & 0.064200 & 8016.50 & 0.591600 & 14000.50 & 0.240500\end{array}$

$20000.50 \quad 0.07380026000 .55 \quad 0.029900$

imp:n $\quad 1.00000 \quad 59 \mathrm{r} \quad 0.00000 \$ 5,139$

$\mathrm{mt} 236$ lwtr.01t

$\mathrm{mt} 2$ lwtr.01t

print 406080100110126

prdmp j 300

kcode 20001.20300

ksrc 9.70 .20 . $-9.70 .085 .09 .7-9.7150 .0$

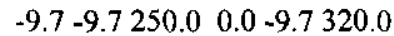

imk4r.1n

Basic model for MCO loaded with 5 fuel baskets.

c All fuel baskets loaded with 20.9 in mk la assemblies.

c Revised 1.25" basket base plates for all baskets.

c fuel basket containing intact fuel assemblies

$\begin{array}{llllllllll}2 & 0 & -17 & 18 & -19 & 20 & -21 & 22 & u=8 & \text { lat }=2\end{array}$ fill $=-5: 5 \quad-5: 5 \quad 0: 0$

$\begin{array}{lllllllllllll}2 & 2 & 2 & 2 & 2 & 2 & 2 & 2 & 2 & 2 & 2 & \$ & 1\end{array}$

$\begin{array}{lllllllllllll}2 & 2 & 2 & 2 & 2 & 2 & 3 & 3 & 3 & 2 & 2 & \$ & 2\end{array}$

$\begin{array}{lllllllllllll}2 & 2 & 2 & 2 & 3 & 3 & 3 & 3 & 3 & 3 & 2 & \$ 3\end{array}$

$\begin{array}{lllllllllllll}2 & 2 & 2 & 3 & 3 & 3 & 3 & 3 & 3 & 3 & 2 & \$ & 4\end{array}$

$\begin{array}{lllllllllllll}2 & 2 & 3 & 3 & 3 & 3 & 3 & 3 & 3 & 3 & 2 & \$ 5\end{array}$

$\begin{array}{llllllllllllll}2 & 2 & 3 & 3 & 3 & 2 & 3 & 3 & 3 & 2 & 2 & \$ 6 & \text { level } 1\end{array}$

$\begin{array}{lllllllllllll}2 & 3 & 3 & 3 & 3 & 3 & 3 & 3 & 3 & 2 & 2 & \$ 7\end{array}$

$\begin{array}{lllllllllllll}2 & 3 & 3 & 3 & 3 & 3 & 3 & 3 & 2 & 2 & 2 & \$ 8\end{array}$

$\begin{array}{lllllllllllll}2 & 3 & 3 & 3 & 3 & 3 & 3 & 2 & 2 & 2 & 2 & \$ 9\end{array}$

$\begin{array}{llllllllllll}2 & 2 & 3 & 3 & 3 & 2 & 2 & 2 & 2 & 2 & 2 & \$ 10\end{array}$

$\begin{array}{llllllllllll}2 & 2 & 2 & 2 & 2 & 2 & 2 & 2 & 2 & 2 & 2 & \$ 11\end{array}$

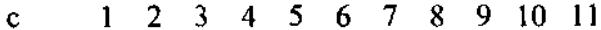


c Mark 4 scrap optimized lattice

$\begin{array}{ccccc}5 & 0 & -1112-13 & 14-1516 & \text { fill }=4 \mathrm{u}=1 \text { lat=2 } \\ 6 & 5 & -18.8200-5 & \mathrm{u}=4 & \text { \$ scrap hex } \\ 7 & 10 & -6.490005-6 & \mathrm{u}=4 & \text { \$ scrap clad } \\ 8 & 236 & -1.000006 & \mathrm{u}=4 & \text { \$ scrap cell }\end{array}$

c Scrap basket

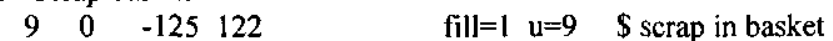

$\begin{array}{lllllll}11 & 236 & -1.00 & -127 & -122 & \mathrm{u}=9 & \text { \$ pipe interior }\end{array}$

$\begin{array}{lllllll}13 & 20 & -8.03 & -122 & 127 & -125 & \mathrm{u}=9\end{array}$ \$ basket base

$\begin{array}{lllllll}14 & 20 & -8.03 & 125 & -126 & \mathrm{u}=9 & \$ \text { basket side }\end{array}$

$\begin{array}{llllll}15 & 236 & -1.00 & 126 & \mathrm{u}=9 & \text { \$ outside basket }\end{array}$

c fictitious water cell for fuel basket lattice

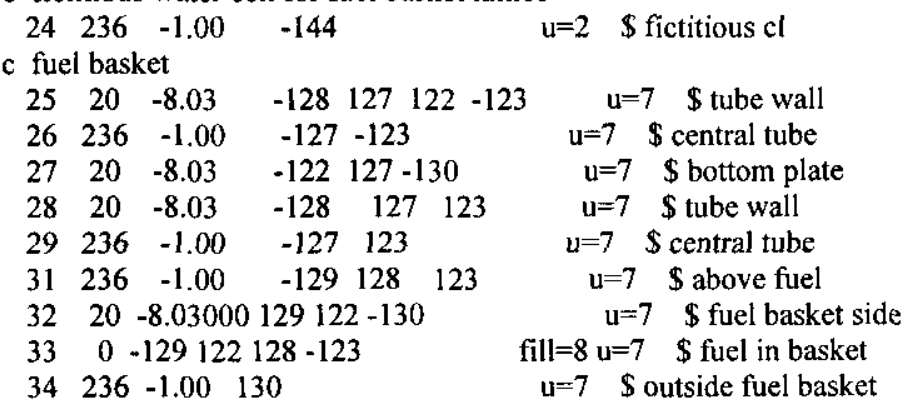

c fuel and scrap basket stack

$\begin{array}{llllll}51 & 0 & -149 & \text { fill=7 } \quad u=11 \quad \$ \text { fuel basket }\end{array}$

$52 \quad 0 \quad 146-149 \# 51 \quad$ trcl $=2$ fill $=7 \quad \mathrm{u}=11 \quad \$$ fuel basket

$53 \quad 0 \quad 146-149 \# 52 \quad$ trcl $=3$ fill $=7 \quad u=11 \quad \$$ fuel basket

$54 \quad 0 \quad 146-149 \# 53 \quad$ tr $\mathrm{cl}=4$ fill $=7 \quad \mathrm{u}=11 \quad \$$ fuel basket

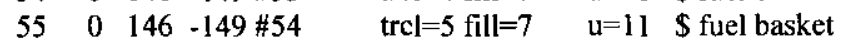

$59 \quad 236-1.000 \quad 152 \# 55 \quad \mathrm{u}=11 \quad \$$ above top basket

c MCO and baskets

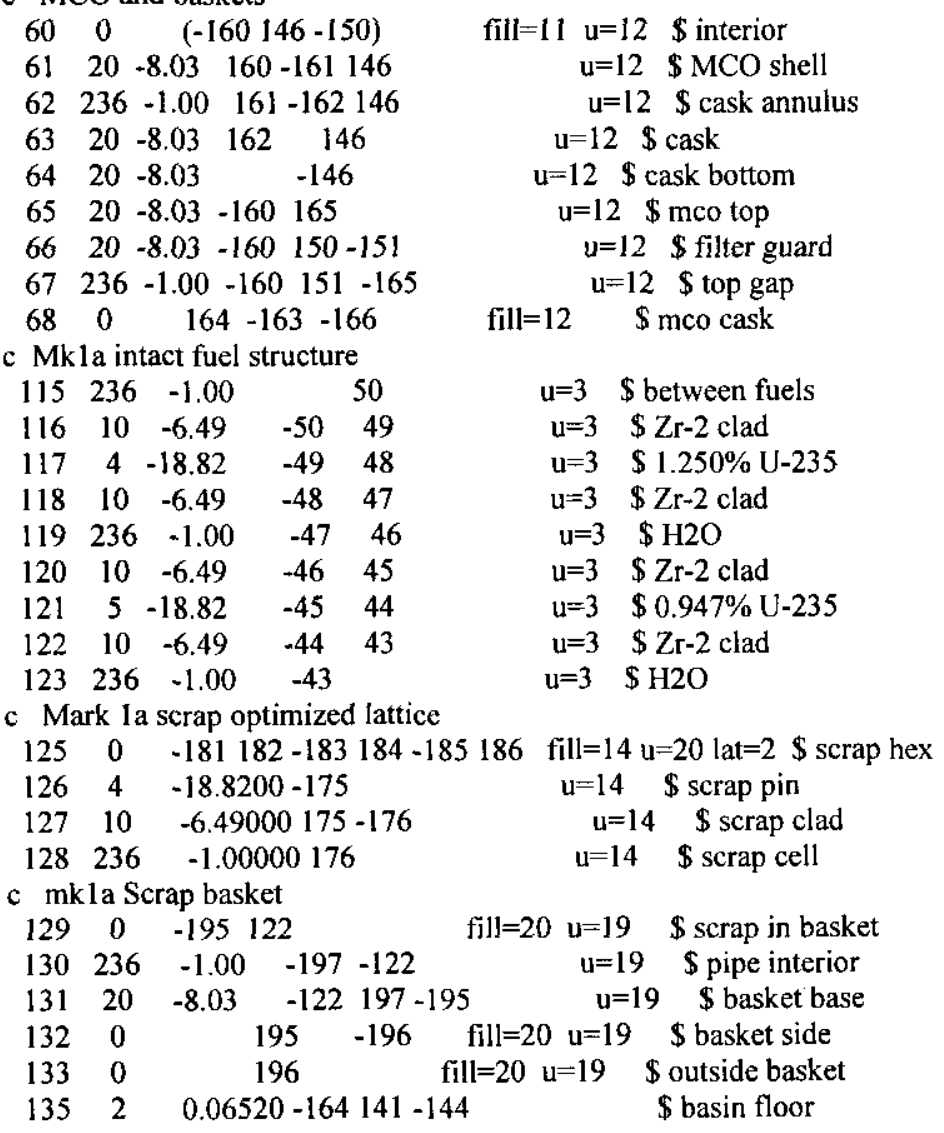




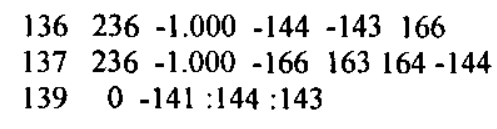

$-141 \cdot 144: 143$

$\begin{array}{lll}5 & \mathrm{cz} & 0.8000000\end{array}$

$\begin{array}{lll}6 & \mathrm{cz} & 0.8572000\end{array}$

$11-p-0.8660254$

$12 \quad \mathrm{p} \quad 0.866025$

13 p 0.866025

14 p 0.8660254

15 py 1.3992000

16 py -1.3992000

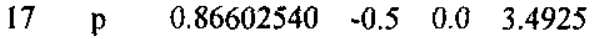

$\begin{array}{llllll}18 & \text { p } & 0.86602540 & -0.5 & 0.0 & -3.4925\end{array}$

$\begin{array}{llllll}19 & \mathrm{p} & 0.86602540 & 0.5 & 0.0 & 3.4925\end{array}$

$\begin{array}{llllll}20 & \text { p } & 0.86602540 & 0.5 & 0.0 & -3.4925\end{array}$

21 py 3.4925

22 py -3.4925

$\begin{array}{llll}43 & \mathrm{cz} & 0.5588 & \$ \mathrm{H} 2 \mathrm{O}\end{array}$

$44 \quad \mathrm{cz} \quad 0.6223 \quad \$ \mathrm{Zr}-2 \mathrm{clad}$

$\begin{array}{llll}45 & \mathrm{cz} & 1.4808 & \$ \text { fuel }\end{array}$

$\begin{array}{llll}46 & \mathrm{cz} & 1.5824 & \$ \mathrm{Zr}-2 \mathrm{clad}\end{array}$

$47 \quad \mathrm{cz} \quad 2.2441 \quad \$ \mathrm{H} 2 \mathrm{O}$

$48 \quad \mathrm{cz} \quad 2.3076 \quad \$ Z \mathrm{Zr}-2 \mathrm{clad}$

$49 \mathrm{cz} \quad 2.9896 \quad$ \$ fuel

$50 \quad \mathrm{cz} \quad 3.0531 \quad \$ \mathrm{Zr}-2 \mathrm{clad}$

122 pz 0.0000000

123 pz 53.086000

c scrap basket side

$\begin{array}{lll}125 & \text { cz } & 28.79725000\end{array}$

126 cz 29.08751997

c process tube pipe

$\begin{array}{lll}127 & \mathrm{cz} & 2.2225000\end{array}$

$128 \quad \mathrm{cz} \quad 3.4925000$

c fuel basket side

$129 \quad \mathrm{cz} \quad 28.41625$

$\begin{array}{lll}130 \mathrm{cz} & 28.73375\end{array}$

141 pz -113.67300

$143 \quad \mathrm{pz} \quad 900.00000$

$144 \quad \mathrm{cz} \quad 500.00000$

$146 \quad \mathrm{pz} \quad-3.1750000$

149 pz 67.68338

$150 \quad \mathrm{pz} \quad 353.83470$

151 pz 357.64470

152 pz 351.11690

c $\mathrm{MCO}$

$\begin{array}{lll}160 \quad \mathrm{cz} & 29.2100\end{array}$

$161 \mathrm{cz} \quad 30.4800$

$162 \quad \mathrm{cz} \quad 32.0004$

$163 \quad \mathrm{cz} \quad 51.0540$

$164 \mathrm{pz} \quad-8.2804$

165 pz 375.000

166 pz 398.1196

175 cz 0.6500000

$176 \mathrm{cz} \quad 0.7107000$

$181 \quad$ p 0.8660254

182 p 0.8660254

183 p 0.8660254

184 p 0.8660254

185 py 1.2013000

186 py -1.2013000

c mk la scrap basket side
\$ water above $\mathrm{MCO}$

$\$$ surrounding water

$\$$ outside world

$\begin{array}{rr}0.0000000 & 1.3992000 \\ 0.0000000 & -1.3992000 \\ 0.0000000 & 1.3992000 \\ 0.0000000 & -1.3992000\end{array}$

$\begin{array}{ll}0.0000000 & 1.3992000\end{array}$ $\begin{array}{rrr}-0.5000000 & 0.0000000 & 1,2013000 \\ -0.5000000 & 0.0000000 & -1.2013000 \\ 0.5000000 & 0.0000000 & 1.2013000 \\ 0.5000000 & 0.0000000 & -1.2013000\end{array}$ 
$195 \quad$ cz $\quad 28.77001997$

196 cz 29.08751997

c process tube pipe

$197 \quad \mathrm{cz} \quad 2.2225000$

$198 \mathrm{cz} \quad 3.4925000$

tr2 $\quad 0.00 \quad 0.00 \quad 70.858380$

$\begin{array}{lllll}\text { tr } 3 & 0.00 & 0.00 & 141.71676\end{array}$

$\begin{array}{lllll}\text { tr4 } & 0.00 & 0.00 & 212.57514\end{array}$

$\begin{array}{lllll}\text { tr5 } & 0.00 & 0.00 & 283.43352\end{array}$

mode $\mathrm{n}$

$\begin{array}{llllll}\mathrm{m} 4 & 92235.50 & -0.01250 & 92238.50 & -0.98750\end{array}$

$\begin{array}{llllll}\mathrm{m} 5 & 92235.50 & -0.00947 & 92238.50 & -0.99053\end{array}$

$\$$ outer fuel

$\mathrm{m} 2361001.50 \mathrm{c} 0.6667008016 .50 \mathrm{c} 0.333300$

$\$$ inner fuel

$\mathrm{ml} 0 \quad 40000.50 \mathrm{c}-1.000000$

$\mathrm{m} 2026000.55 \mathrm{c} 67.97000124000 .50 \mathrm{c} 20.00000028000 .50 \mathrm{c} 10.000000$

$25055.50 \mathrm{c} 2.0000006000 .50 \mathrm{c} 0.030000$

m2 $\quad 1001.50 \quad 0.0642008016 .50 \quad 0.59160014000 .50 \quad 0.240500$

$\begin{array}{llll}20000.50 & 0.073800 & 26000.55 & 0.029900\end{array}$

imp:n $\quad 1.00000 \quad 55 \mathrm{r} \quad 0.00000 \$ 5,139$

$\mathrm{mt} 236$ lwtr.01t

$\mathrm{mt2}$ lwtr.01t

print 406080100110126

prdmp j 300

kcode 2000 1. 20300

ksrc 9.70 .20 . $-9.70 .085 .09 .7-9.7150 .0$

$\begin{array}{llll}-9.7 & -9.7250 .0 & 0.0 & -9.7320 .0\end{array}$ 


\section{TECHNICAL PEER REVIEWS}

\section{CHECKLIST FOR TECHNICAL PEER REVIEW}

Document Reviewed:

Title: Complete Misloading of a Mark IV MCO with Mark IA Fuel (SNF-4848, Rev 0)

Author: S. F. Kessler

Date: $7 / 19 / 99$

Scope of Review: Full document

Yes No* NA

[ ] [ ] [ X]**Previous reviews complete and cover analysis, up to scope of this review, with no gaps.

[X] [ ] [ ] Problem completely defined.

[ ] [ ] [ X] Accident scenarios developed in a clear and logical manner.

[X] [ ] [ ] Necessary assumptions explicitly stated and supported.

[X] [ ] [ ] Computer codes and data files documented.

[X] [ ] [ ] Data used in calculations explicitly stated in document.

[X] [ ] [ ] Data checked for consistency with original source information as applicable.

[ ] [ ] [X] Mathematical derivations checked including dimensional consistency of results.

[X] [ ] [ ] Models appropriate and used within range of validity or use outside of range of established validity justified.

[X] [ ] [ ] Hand calculations checked for errors. Spreadsheet results should be treated exactly the same as hand calculations.

[X] [ ] [ ] Software input correct and consistent with document reviewed.

[X] [ ] [ ] Software output consistent with input and with results reported in document reviewed.

[X] [ ] [ ] Limits/criteria/guidelines applied to analysis results are appropriate and referenced.

Limits/criteria/guidelines checked against references.

[X] [ ] [ ] Safety margins consistent with good engineering practices.

[X] [ ] [ ] Conclusions consistent with analytical results and applicable limits.

[X] [ ] [ ] Results and conclusions address all points required in the problem statement.

[X] [ ] [ ] Format consistent with applicable guides or other standards.

[ ] [ ] [X]**Review calculations, comments, and/or notes are attached.

[X] [ ] [ ] Traceability

[X] [ ] [ ] Document approved (for example, the reviewer affirms the technical accuracy of the document).

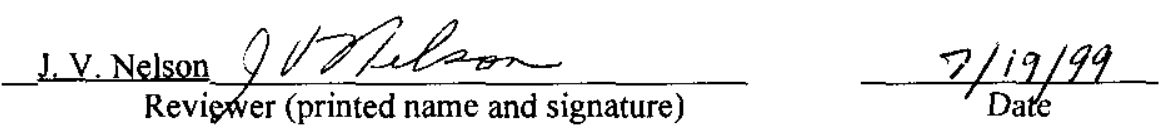

* All "no" responses must be explained below or on an additional sheet.

** Any calculations, comments, or notes generated as part of this review should be signed, dated, and attached to this checklist. Such material should be labeled and recorded in such a manner as to be intelligible to a technically qualified third party. 


\section{DISTRIBUTION SHEET}

\begin{tabular}{|c|c|c|c|c|c|}
\hline $\begin{array}{l}\text { To } \\
\text { Distribution }\end{array}$ & \multicolumn{3}{|c|}{$\begin{array}{l}\text { From } \\
\text { Nuclear Safety }\end{array}$} & \multicolumn{2}{|l|}{$\begin{array}{l}\text { Page } 1 \\
\end{array}$} \\
\hline \multirow{2}{*}{\multicolumn{4}{|c|}{$\begin{array}{l}\text { Project Title / Work Order } \\
\text { HNF-4848, Rev } 0 \\
\text { Complete Misloading of a Mark IV MCO with Mark IA Fuel }\end{array}$}} & \multirow{2}{*}{\multicolumn{2}{|c|}{ EDT No. 626887}} \\
\hline & & & & & \\
\hline Name & MSIN & $\begin{array}{l}\text { Text with } \\
\text { All Attach. }\end{array}$ & $\begin{array}{l}\text { Text } \\
\text { Only }\end{array}$ & $\begin{array}{l}\text { Attach/Appendix } \\
\text { Only }\end{array}$ & EDT/ECN Only \\
\hline Sixto Almodovar & A3-02 & & & & \\
\hline R. D. Crowe & R3-26 & & & & \\
\hline L. J. Garvin & R3-26 & & & & \\
\hline K. D. Gibson & R3-26 & & & & \\
\hline S. B. Harrington (5) & R3-26 & & & & \\
\hline S. F. Kessler & R3-26 & & & & \\
\hline C. T. Miller & $\mathrm{X3-79}$ & & & & \\
\hline J. V. Nelson & R3-26 & & & & \\
\hline S. H. Peck & R3-26 & & & & \\
\hline C. A. Thompson & R3-86 & & & & \\
\hline H. Toffer & B4-44 & & & & \\
\hline SNF Project Files & R3-11 & & & & \\
\hline
\end{tabular}

